\title{
The effect of deafness on the physical fitness parameters of elite athletes
}

\author{
Bihter Akınoğlu,**, Tuğba Kocahan ${ }^{2}$ \\ 'Department of Physiotherapy and Rehabilitation, Faculty of Health Sciences, Ankara Yıldırım Beyazıt University, Ankara, Turkey \\ ${ }^{2}$ Department of Health Services, Center of Athlete Training and Health Research, Sports General Directorship, The Ministry of Youth and Sports, Ankara, Turkey
}

The purpose of this study is to determine the health-related physical fitness parameters of athletes, who are deaf. The study group consisted of 137 deaf athletes ( 52 females and 85 males with a mean age of $23.99 \pm 6.56$ years) who have participated in the 2017 Deaflympics games. The assessed parameters included the isokinetic muscle strength, core endurance, balance assessments, grip strength, and body composition. At the end of the study, the following results were obtained. Hamstring/quadriceps ratio was in the range of $53 \%-54 \%$, which was within $50 \%-60 \%$ of the normal limit. Endurance of the anterior and posterior core muscles was low as compared to the normative data. The athletes' balance was better with their eyes open than closed; and the grip strength was low as compared to the normative data in the literature. Also, the body fat percentage of deaf female athletes were similar to ones in healthy individuals with sedentary lifestyle, whereas male ones had slightly lower body fat mass percentage. These results can be used as the initial reference parameters to define the training program requirements of deaf athletes.

Keywords: Muscle strength, Balance, Core stability, Body composition, Disability, Health

\section{INTRODUCTION}

In recent years, there have been many noticeable attempts to prevent individuals with disabilities from being separated from the society and living an isolated life. One of the most important and effective ways is organizing competitive sporting events adapted for these individuals. Athletes with hearing impairment do not experience any physical deprivation and can participate in the competitive sports without any specific limitations. The communicative barriers associated with deafness are the only factors that distinguish them from other athletes (Kurková et al., 2011). These barriers often make them to live in their own bubble and ultimately isolate them from the society. Participation in specialized sports helps them to transform and also to become social individuals even for the rest of their lives (Dursun et al., 2015; Stewart and Ellis, 2005). Athletes with hearing impairments compete in the Deaflympics, which is held every 4 years. It is organized a year after the Olympic Games and is not part of the Paralympic Games.
Even though the Paralympic Games have less history than the Deaf Olympics, a lot more research have been conducted related to the physical activities and characteristics of Paralympic athletes (Kurková et al., 2011).

One of the most important parameters to be investigated in deaf athletes with a lack of sensory input due to loss of hearing ability is the working capacity, which is also defined as physical fitness. The physical fitness of an athlete has many components including the physiological and motor performance. The subcomponents can be listed as the cardio-respiratory endurance, muscle strength, speed, flexibility, agility, balance, reaction time and body composition (Jackson, 2006). These components, at the same time, are also defined as health and sports performance related physical fitness parameters, since they are related to each other. There are numerous physical fitness assessment tests which include components that are not only limited to strength and endurance, but also include speed and agility. These tests help to gather important data for a specific group of population.
*Corresponding author: Bihter Akınoğlu (iD https://orcid.org/0000-0002-8214-7895 Department of Physiotherapy and Rehabilitation, Faculty of Health Sciences,

Ankara Yildırım Beyazıt University, Ankara, Turkey

E-mail: rgkardelen@yahoo.com

Received: March 3, 2019 / Accepted: April 11, 2019
This is an Open Access article distributed under the terms of the Creative Commons Attribution Non-Commercial License (http://creativecommons.org/licenses/by-nc/4.0/) which permits unrestricted non-commercial use, distribution, and reproduction in any medium, provided the original work is properly cited. 
Studies on deaf athletes are extremely limited. Therefore, many additional studies are needed to shed light on the physical characteristics of these athletes. In a way, the studies on the health-related parameters of deaf athletes can be pioneering. We suggest that the parameters identified in our study can be used as an initial reference to set the requirements of the training program for deaf athletes. Because, these results can contribute to the creation of new methods and ideas on the development of optimal training programs to improve to athletes' performance and competitiveness.

\section{MATERIALS AND METHODS}

This study was conducted on 137 elite deaf athletes, who have applied to our Sports Education and Health Research Center. Descriptive characteristics of the athletes are given in Table 1.

The inclusion criteria for this study were as follows; not having any mental problems, to have no disability other than hearing impairment, to be a volunteer for the study and to have participated in an international competition for at least 3 years. The purpose and the context were explained to the athletes prior to the study. The age, height, weight, dominant side, and years of experience of the athletes, who met the study criteria, were recorded. Isokinetic knee strength test, static and dynamic core endurance tests, bal- ance evaluation, grip strength, and body composition measurement were performed as a part of the evaluations. The communication took place by the help of sign language interpreters from the athletes' own teams. All tests were performed with an athletic outfit and without the use of any hearing aids, as they are generally not allowed during competitions. The procedures for this study were approved by the Ethics Committee (2017-593-31). All fitness assessment procedures were completed in 2 days. During the first day of the assessment, body composition was evaluated in the morning before the athletes had breakfast. Then, grip strength was evaluated in the afternoon with full stomach. During the second day of the assessment, balance and isokinetic strength were evaluated in the morning and core endurance in the afternoon. Every assessment was performed by a physiotherapist with a minimum 3 years of experience in his/her own department.

\section{Evaluation}

\section{Evaluation of isokinetic muscle strength}

Isokinetic strength evaluation was performed with the IsoMed 2000 (D. \& R. Ferstl GmbH, Hemau, Germany) device. Athletes were asked to jog for $10 \mathrm{~min}$ before starting the test as a warm-up. After the isokinetic device's measurement settings were automatically adjusted, the athlete was seated on the device. Additional adjustments were made where necessary to position the pivot

Table 1. Descriptive features of athletes

\begin{tabular}{|c|c|c|c|c|}
\hline Variable & All athletes $(n=137)$ & Female athletes ( $n=52)$ & Male athletes $(n=85)$ & $P$-value ${ }^{\text {a) }}$ \\
\hline Age (yr) & $23.99 \pm 6.56(15-44)$ & $22.05 \pm 5.84$ & $25.09 \pm 6.80$ & 0.005 \\
\hline Height (cm) & $1.71 \pm 0.08(1.48-1.88)$ & $1.64 \pm 0.06$ & $1.73 \pm 0.20$ & 0.000 \\
\hline Weight (kg) & $69.53 \pm 15.54(48-114)$ & $58.21 \pm 10.51$ & $76.09 \pm 13.68$ & 0.000 \\
\hline Body mass index $\left(\mathrm{kg} / \mathrm{m}^{2}\right)$ & $23.59 \pm 4.37(17.03-35.42)$ & $21.42 \pm 3.93$ & $24.47 \pm 4.65$ & 0.000 \\
\hline Sport year & $9.36 \pm 6.14(3-36)$ & $8.13 \pm 6.13$ & $10.15 \pm 6.05$ & 0.031 \\
\hline \multicolumn{5}{|l|}{ Sport type, n } \\
\hline Karate & 31 & 12 & 19 & \\
\hline Taekwondo & 16 & 6 & 10 & \\
\hline Free style wrestling & 12 & 0 & 12 & \\
\hline Greco-Roman wrestling & 15 & 0 & 15 & \\
\hline Tennis & 10 & 4 & 6 & \\
\hline Running & 10 & 2 & 8 & \\
\hline Basketball & 17 & 17 & 0 & \\
\hline Swimming & 9 & 4 & 5 & \\
\hline Judo & 17 & 7 & 10 & \\
\hline \multicolumn{5}{|l|}{ Gender, n (\%) } \\
\hline Female & $52(38)$ & & & \\
\hline Male & $85(62)$ & & & \\
\hline
\end{tabular}

Values are presented as mean \pm standard deviation (range) unless otherwise indicated. allndependent samples t-test. 
point into the lateral femoral condyle. The gravitational effect was eliminated. The evaluation protocol included 5 repetition knee flexion/extension strength assessments at $60 \%$ sec angular velocities. Immediately prior to the muscle strength test, 3 repetitive warmups were included in the protocol to help the athletes rehearse the movement. During the evaluations, the visual feedback of the isokinetic device was provided and explained to the athletes. The evaluations were performed bilaterally. The assessment started with the dominant side followed by a 3-min rest and completed with the nondominant side (Fischer et al., 2018). The peak torque (PT) and peak torque/weight ratio (PT/W) was recorded. Then, percentage of flexor/extensor muscle strength and percentage of dominant/nondominant side (right/left) muscle strength were calculated.

\section{Evaluation of static and dynamic core endurance}

Static core endurance was evaluated with the static trunk flexion endurance test, the static trunk extension endurance test, and the lateral bridge endurance test, in which the results were recorded in seconds (Cowan, 2012).

Flexor endurance test: Athletes were positioned lying supine with the knees and hips flexed $90^{\circ}$. Their feet were stabilized by the physiotherapist and they crossed their arms across the chest, placing their hands on opposite shoulders. After the angulus inferior of scapulae had been lifted from the examination table, the test was started and the duration to maintain the position was recorded.

Extension endurance test: Athletes were positioned lying prone with their upper body (only above the level of the anterior superior iliac crest) off the surface of the examiner table. Their arms were crossed across the chest and hands were placed on the opposite shoulders. Once their feet were stabilized and they were in complete horizontal position, the test was started, in which the duration to maintain the horizontal position was recorded.

Lateral bridge test: The assessment started with the dominant side followed by a 3-min rest and completed with the nondominant side. Athletes were positioned lying on their side with their knees extended with the foot on top placed in front of the lower foot, and the hip lifted off the table. The body weight was supported on the lower elbow and feet. The duration to maintain a straight body posture was recorded.

Dynamic endurance tests: This category included the sit up test, the chest raise test and the lateral flexion (right-left) test, all of which involve the similar static core endurance test positions, and the 1-min timing was used to assess the strength of the core strength. The number of repetitions the athletes were able to per- form within a 1-min timing were recorded (McIntosh et al., 1998).

\section{Evaluation of balance}

Balance evaluation was tested with the Human Body Equilibrium 360 device (HUBER360) (http://international.chattgroup. com/sites/default/files/user_guide_huber_360_1786.pdf). Balance measurements included the stability assessments, evaluation of single leg balance and functional reach test. For the stability evaluation, the duration was set as 50 sec during which the athletes were tested how long they were able to maintain the posture with their eyes closed and open, the length and area which athletes draw for balance strategies during the test were recorded. For the single leg balance assessment, one foot was placed in a specific position and the other leg was elevated until the thigh was parallel to the ground. Then, the athletes were instructed to maintain their balance for a 300-sec period, and the aberration from the initial point was recorded. The amount of maximal reach in each direction was determined in the functional reach test. During the course of the test, athletes were instructed not to bend their body and not to lose contact of the bottom of their feet and toes from the ground (Akınoğlu and Kocahan, 2018).

\section{Evaluation of grip strength}

The grip strength of the athletes was tested by the Jamar hand dynamometer (Bohannon et al., 2006). First, each athlete was described how the test was performed by showing the measurement instrument and the correct gripping position. Measurements has started with the right hand's grip strength. Three repetitions of grip were performed while athletes were sitting with their shoulders adducted, the elbows flexed at $90^{\circ}$ and the forearm held in neutral position. Each measurement was taken alternately between the right and left hands to reduce the effect of fatigue. Minimum of 15-sec breaks were taken between each measurements and the test results were recorded in kilograms. Averages of three measurements were taken to determine the final test result (Bohannon et al., 2006).

\section{Evaluation of body composition}

Athletes' body composition was measured with a Tanita Body Composition Analyzer (TBF 300 M) (Tanita Corporation of America, Inc., www.tanita.com). Athletes were asked not to perform any intense physical activities and not to consume any diuretic drinks such as tea and coffee within $24 \mathrm{hr}$ prior to the tests. Athletes who did not comply with these requirements were excluded from the study. The measurements were taken barefoot. For the 
measurements, the athletes were asked to step on the metal electrodes, to stand upright and to hold the hand electrodes. Any metal accessories (watches, rings, necklaces, etc.) were removed during the tests. The body composition parameters recorded as a part of this evaluation consist of fat free mass ( $\mathrm{kg}$ ), fat mass (FM) $(\mathrm{kg})$, and fat mass percentage (FM\%) (Nuñez et al., 1997).

\section{Statistical analysis}

Descriptive statistical analysis of all collected variables was done using IBM SPSS Statistics ver. 22.0 (IBM Co., Armonk, NY, USA). The results were expressed as mean \pm standard deviation for all male and female athletes. Kolmogorov-Smirnov test, which was developed specifically for a large number of data, was used to determine whether our data was normally distributed or not. The data pairs that were not normally distributed were analyzed by the Wilcoxon test, whereas the data pairs that were normally dis-

Table 2. Mean values of knee flexion and extension's PT

\begin{tabular}{|c|c|c|c|}
\hline Muscle strength & Dominant side & Nondominant side & $P$-value \\
\hline \multicolumn{4}{|l|}{ All athletes $(n=137)$} \\
\hline Knee Flexion PT (N/m) & $97.60 \pm 32.44$ & $96.67 \pm 32.01$ & 0.73 \\
\hline Knee Flexion PT/W (Nm/kg) & $1.38 \pm 0.29$ & $1.37 \pm 0.31$ & 0.62 \\
\hline Knee Extension PT (N/m) & $181.29 \pm 54.28$ & $177.96 \pm 57.35$ & 0.62 \\
\hline Knee Extension PT/W (Nm/kg) & $2.58 \pm 0.46$ & $2.54 \pm 0.56$ & 0.16 \\
\hline Knee Flexion/Extension \% & $53.88 \pm 8.11$ & $54.96 \pm 9.92$ & 0.68 \\
\hline Knee Flexion DM/NDM \% & \multicolumn{2}{|c|}{$102.00 \pm 15.23$} & 0.74 \\
\hline Knee Extension DM/NDM\% & \multicolumn{2}{|c|}{$104.15 \pm 21.41$} & \\
\hline \multicolumn{4}{|l|}{ Female athletes $(n=52)$} \\
\hline Knee Flexion PT (N/m) & $69.18 \pm 13.76$ & $68.19 \pm 13.37$ & 0.30 \\
\hline Knee Flexion PT/W (Nm/kg) & $1.19 \pm 0.21$ & $1.17 \pm 0.22$ & 0.33 \\
\hline Knee Extension PT (N/m) & $132.09 \pm 23.94$ & $130.09 \pm 23.47$ & 0.07 \\
\hline Knee Extension PT/W (Nm/kg) & $2.29 \pm 0.36$ & $2.24 \pm 0.40$ & 0.06 \\
\hline Knee Flexion/Extension \% & $52.54 \pm 8.11$ & $52.79 \pm 7.83$ & 0.51 \\
\hline Knee Flexion DM/NDM \% & \multicolumn{2}{|c|}{$102.21 \pm 12.42$} & 0.53 \\
\hline Knee Extension DM/NDM\% & \multicolumn{2}{|c|}{$102.84 \pm 12.22$} & \\
\hline \multicolumn{4}{|l|}{ Male athletes $(n=85)$} \\
\hline Knee Flexion PT (N/m) & $116.63 \pm 27.15$ & $115.44 \pm 26.34$ & 0.99 \\
\hline Knee Flexion PT/W (Nm/kg) & $1.51 \pm 0.26$ & $1.50 \pm 0.28$ & 0.99 \\
\hline Knee Extension PT (N/m) & $213.22 \pm 44.02$ & $209.49 \pm 50.88$ & 0.60 \\
\hline Knee Extension PT/W (Nm/kg) & $2.78 \pm 0.42$ & $2.74 \pm 0.56$ & 0.53 \\
\hline Knee Flexion/Extension \% & $54.77 \pm 8.04$ & $56.40 \pm 10.90$ & 0.30 \\
\hline Knee Flexion DM/NDM \% & \multicolumn{2}{|c|}{$101.88 \pm 16.90$} & 0.25 \\
\hline Knee Extension DM/NDM\% & \multicolumn{2}{|c|}{$105.03 \pm 25.78$} & \\
\hline
\end{tabular}

Values are presented as mean \pm standard deviation.

PT, peak torque; W, weight; DM, dominant side; NDM, nondominant side.

PTM: percentage of flexor/extensor muscle strength and percentage of dominant side/nondominant (right/left) muscle strength and comparison of dominant and nondominant sides at $60 \%$ sec.

alPaired samples $t$-test. tributed were analyzed by the Paired Samples $t$-test. Both tests help to determine if there was any difference between the dominant and nondominant side. The level of significance was accepted as $P<0.05$.

\section{RESULTS}

Table 2 shows the athletes' mean values of knee flexion and extension PT, PT/W, percentage of flexion/extensor muscle strength, percentage of dominant side/nondominant (right/left) muscle strength and comparison of the dominant and nondominant sides at $60^{\circ} / \mathrm{sec}$ angular velocity. The mean values of core endurance and core strength tests are shown in Table 3; the average balance values are shown in Tables 4 and 5; the average grip strength values are shown in Table 6; and the mean values of body FM\%, FM, and lean body mass of athletes are shown in Table 7 .

\section{DISCUSSION}

The literature contains many studies on athletes engaged in different branches of sports, but athletes with disabilities have been neglected. The purpose of this study was to determine muscle strength, static and dynamic core endurance, balance evaluation, grip strength and body composition, which are some of the physical fitness parameters of athletes, who are deaf. The results of our study showed that, isokinetic muscle strength ratio for Hamstring/Quadriceps was in the range of 53\%-54\% and the dominant/nondominant side ratio was less than $5 \%$ for all athletes. Particularly in male athletes, endurance of the anterior and posterior core muscles was low. Both group of athletes' balance with

Table 3. Mean values of static and dynamic core endurance test result

\begin{tabular}{lrrr}
\hline & $\begin{array}{c}\text { All athletes } \\
(\mathrm{n}=137)\end{array}$ & $\begin{array}{c}\text { Female ath- } \\
\text { letes }(\mathrm{n}=52)\end{array}$ & $\begin{array}{c}\text { Male athletes } \\
(\mathrm{n}=85)\end{array}$ \\
\hline Static core endurance tests $(\mathrm{sec})$ & & & \\
$\quad$ Flexor endurance test & $100.61 \pm 66.83$ & $109.69 \pm 86.32$ & $95.42 \pm 52.47$ \\
Extensor endurance test & $116.11 \pm 55.34$ & $126.06 \pm 63.19$ & $110.42 \pm 49.83$ \\
Lateral flexion (dominant) & $81.59 \pm 41.47$ & $73.01 \pm 38.11$ & $86.49 \pm 42.73$ \\
Lateral flexion (nondominant) & $82.24 \pm 43.41$ & $72.60 \pm 43.39$ & $87.74 \pm 42.70$ \\
Dynamic core endurance tests (repetition) & & \\
Sit-up & $49.29 \pm 12.34$ & $43.20 \pm 10.31$ & $52.55 \pm 12.15$ \\
Chest raise & $45.53 \pm 18.18$ & $34.77 \pm 13.41$ & $51.36 \pm 17.82$ \\
Lateral flexion (dominant side) & $68.41 \pm 31.60$ & $48.65 \pm 21.49$ & $79.01 \pm 31.15$ \\
Lateral flexion (nondominant side) & $70.87 \pm 32.61$ & $52.72 \pm 26.95$ & $80.49 \pm 31.35$ \\
Back extensor test & $68.32 \pm 21.06$ & $59.00 \pm 19.86$ & $73.40 \pm 20.06$ \\
\hline
\end{tabular}

Values are presented as mean \pm standard deviation. 
Table 4. Mean values of stability and functional reach tests of balance evaluation

\begin{tabular}{|c|c|c|c|}
\hline Test & All athletes $(n=137)$ & Female athletes $(n=52)$ & Male athletes $(n=85)$ \\
\hline \multicolumn{4}{|l|}{ Stability test } \\
\hline \multicolumn{4}{|l|}{ Length (mm) } \\
\hline Eyes opened & $672.73 \pm 271.05$ & $738.22 \pm 316.20$ & $632.66 \pm 232.30$ \\
\hline Eyes closed & $886.65 \pm 258.95$ & $908.94 \pm 249.94$ & $873.02 \pm 264.84$ \\
\hline \multicolumn{4}{|l|}{ Area $\left(\mathrm{mm}^{2}\right)$} \\
\hline Eyes opened & $399.34 \pm 488.87$ & $416.47 \pm 379.57$ & $388.86 \pm 546.97$ \\
\hline Eyes closed & $517.32 \pm 358.44$ & $536.33 \pm 380.25$ & $505.69 \pm 346.23$ \\
\hline \multicolumn{4}{|l|}{ Speed $(\mathrm{mm} / \mathrm{sec})$} \\
\hline Eyes opened & $13.44 \pm 5.42$ & $14.75 \pm 5.35$ & $12.65 \pm 4.64$ \\
\hline Eyes closed & $17.73 \pm 5.17$ & $18.17 \pm 4.99$ & $17.46 \pm 5.29$ \\
\hline \multicolumn{4}{|l|}{ Functional reach test $\left(\mathrm{mm}^{2}\right)$, direction } \\
\hline $0^{\circ}$ (reach to right side) & $189.70 \pm 27.30$ & $193.48 \pm 27.11$ & $187.38 \pm 27.32$ \\
\hline $45^{\circ}$ (reach obliquely between right and forward) & $158.00 \pm 28.12$ & $163.88 \pm 25.90$ & $154.41 \pm 28.96$ \\
\hline $90^{\circ}$ (reach to forward) & $121.33 \pm 25.32$ & $120.38 \pm 29.94$ & $121.91 \pm 22.20$ \\
\hline $135^{\circ}$ (reach obliquely between left and forward) & $164.34 \pm 33.52$ & $162.32 \pm 36.85$ & $165.57 \pm 31.47$ \\
\hline $180^{\circ}$ (reach to left side) & $180.50 \pm 29.28$ & $180.73 \pm 28.33$ & $180.36 \pm 30.01$ \\
\hline $225^{\circ}$ (reach obliquely between left and back) & $148.66 \pm 30.42$ & $146.53 \pm 27.34$ & $149.96 \pm 32.25$ \\
\hline $270^{\circ}$ (reach to back) & $109.15 \pm 25.62$ & $103.17 \pm 21.88$ & $112.81 \pm 27.13$ \\
\hline $315^{\circ}$ (reach obliquely between right and back) & $158.10 \pm 33.70$ & $154.21 \pm 31.28$ & $160.48 \pm 35.06$ \\
\hline Total & $68,170.30 \pm 15921$ & $67,534.92 \pm 13.370$ & $68,559.00 \pm 17.363$ \\
\hline
\end{tabular}

Values are presented as mean \pm standard deviation.

Table 5. Mean values of single leg balance tests

\begin{tabular}{llll}
\hline Single leg balance & \multicolumn{1}{c}{ Dominant } & Nondominant & $P$-value ${ }^{\mathrm{a})}$ \\
\hline $\begin{array}{l}\text { All athletes }(\mathrm{n}=137) \\
\text { Length }(\mathrm{mm})\end{array}$ & $1,635.00 \pm 498.27$ & $1,573.86 \pm 433.61$ & 0.61 \\
$\quad$ Area $\left(\mathrm{mm}^{2}\right)$ & $1,560.27 \pm 3,087.00$ & $1,790.69 \pm 5,799.57$ & 0.06 \\
Female athletes $(\mathrm{n}=52)$ & & & \\
Length $(\mathrm{mm})$ & $1,749.62 \pm 589.71$ & $1,641.57 \pm 508.56$ & 0.14 \\
Area $\left(\mathrm{mm}^{2}\right)$ & $1,507.93 \pm 2,002.87$ & $1,270.21 \pm 889.69$ & 0.63 \\
Male athletes $(\mathrm{n}=85)$ & & & \\
Length $(\mathrm{mm})$ & $1,564.88 \pm 421.57$ & $1,532.43 \pm 377.90$ & 0.25 \\
Area $\left(\mathrm{mm}^{2}\right)$ & $1,592.30 \pm 3,605.42$ & $2,109.09 \pm 7,328.43$ & 0.83 \\
\hline
\end{tabular}

Values are presented as mean \pm standard deviation.

alPaired samples t-test.

eyes open was better than their eyes closed, and their functional reach back and forward was limited as compared to their functional reach in other directions. The grip strength was also low in both genders. Moreover, percentage of the body fat of deaf female athletes were similar to healthy sedentary people, whereas the male deaf athletes had slightly lower body FM\%. We suggest that the findings in our study shall set the basis for future studies.

A certain level of physical fitness is essential for the performance of sports related activities in many sports branches. Muscle strength, which is one of the physical fitness parameters, is needed
Table 6. Mean values of grip strength

\begin{tabular}{lccc} 
Grip strength $(\mathrm{kg})$ & $\begin{array}{c}\text { All athletes } \\
(\mathrm{n}=137)\end{array}$ & $\begin{array}{c}\text { Female athletes } \\
(\mathrm{n}=52)\end{array}$ & $\begin{array}{c}\text { Male athletes } \\
(\mathrm{n}=85)\end{array}$ \\
\hline Right & $36.67 \pm 11.25$ & $24.82 \pm 4.32$ & $43.51 \pm 7.78$ \\
Left & $35.25 \pm 11.80$ & $22.48 \pm 4.11$ & $42.47 \pm 7.92$ \\
\hline
\end{tabular}

Values are presented as mean \pm standard deviation.

Table 7. Body composition of athletes

\begin{tabular}{lccc}
\hline & $\begin{array}{c}\text { All athletes } \\
(\mathrm{n}=137)\end{array}$ & $\begin{array}{c}\text { Female athletes } \\
(\mathrm{n}=52)\end{array}$ & $\begin{array}{c}\text { Male athletes } \\
(\mathrm{n}=85)\end{array}$ \\
\hline FM\% & $17.05 \pm 6.99$ & $24.50 \pm 4.88$ & $13.88 \pm 5.10$ \\
FM $(\mathrm{kg})$ & $12.3 \pm 6.14$ & $15.12 \pm 5.51$ & $11.11 \pm 6.03$ \\
FFM $(\mathrm{kg})$ & $59.22 \pm 12.15$ & $45.07 \pm 4.68$ & $65.23 \pm 8.91$ \\
\hline
\end{tabular}

Values are presented as mean \pm standard deviation.

FM, fat mass; FM\%, FM percentage; FFM, fat free mass.

to outperform the competitor (Stølen et al., 2005; Ziv and Lidor, 2010). In our study, an isokinetic dynamometer, which is considered to be the gold standard, was chosen to provide an objective and easily interpretable numerical data for assessing muscle strength (Xaverova et al., 2015).

Compared to the free-weight tests, isokinetic dynamometers offer some advantages as they are able to determine the muscle imbalance and PT values at different angular velocities (Cometti 
et al., 2001). In our study, the angular velocity at $60 \% \mathrm{sec}$ was preferred as it has been commonly used in many studies conducted on athletes to determine the isokinetic muscle strength (Berckmans et al., 2017; Undheim et al., 2015; Vieira et al., 2016). In order to increase the objectivity and generalization of the physical fitness parameters, we have included a maximum number of athletes from different sports branches. Each sports branch is composed of different sport-specific activities, which is performed with different primary muscle groups, and require different amounts of muscle power. This led to the necessity of establishing the evaluation protocol with one of the active and commonly used muscle groups in each sports branch included in our study. Consequently, quadriceps and hamstring muscles, which are actively involved in all sports, were included in the isokinetic evaluation protocol. As a result of our study, mean values of knee flexion and extension's PT of dominant and nondominant side were $97.60 \pm 32.44,96.67 \pm$ $32.01 ; 181.29 \pm 54.28,177.96 \pm 57.35 \mathrm{Nm}$, respectively for all athletes, $69.18 \pm 13.76,68.19 \pm 13.37$; 132.09 $\pm 23.94,130.09 \pm$ $23.47 \mathrm{Nm}$ for female athletes, and $116.63 \pm 27.15,115.44 \pm 26.34$; $213.22 \pm 44.02,209.49 \pm 50.88 \mathrm{Nm}$ for male athletes. Mean values of knee flexion and extension's PT/W of dominant and nondominant sides were $1.38 \pm 0.29,1.37 \pm 0.31 ; 2.58 \pm 0.46,2.54 \pm$ $0.56 \mathrm{Nm} / \mathrm{kg}$, respectively for all athletes, $1.19 \pm 0.21,1.17 \pm 0.22$; $2.29 \pm 0.36,2.24 \pm 0.40 \mathrm{Nm} / \mathrm{kg}$ for female athletes, and $1.51 \pm$ $0.26,1.50 \pm 028 ; 2.78 \pm 0.42,2.74 \pm 0.56 \mathrm{Nm} / \mathrm{kg}$ for male athletes. Percentage of dominant/nondominant side muscle strength at a $60^{\circ} / \mathrm{sec}$ angular velocity was determined as $102.00 \pm 15.23$, $104.15 \pm 21.41$ for all athletes; $102.21 \pm 12.42,102.84 \pm 12.22$ for female athletes; and $101.88 \pm 16.90,105.03 \pm 25.78$ for male athletes for flexion and extension, respectively. There are many studies related to the evaluation of isokinetic strength in different sports branches. These studies involve a wide variety of athletes in the branches of handball, volleyball, basketball, soccer, wrestling and judo (Ghrairi et al., 2014; Kurdak et al., 2005; Zi-Hong et al., 2013). However, to our knowledge, there are no studies that focuses on determining muscle strength characteristics of deaf individuals with sedentary lifestyles or any studies in which the athletes of different sports branches without any disability are evaluated together as in our study.

It has been reported in previous studies that the imbalance of ipsilateral (knee flexors/knee extensors) and bilateral (dominant/ nondominant side) ratio may be associated with increased hamstring and other soft tissue injuries (Croisier et al., 2008; Kurdak et al., 2005). In the related literature, ipsilateral ratio is normally accepted to be between $50 \%-60 \%$ at low angular velocities $\left(60^{\circ}-\right.$ $90^{\circ} / \mathrm{sec}$ ) (Cheung et al., 2012). In our study, the hamstring/quadriceps ratio was determined as $53 \%-54 \%$ (dominant side, nondominant side, respectively) for all athletes, $52 \%-52 \%$ for female athletes, and $54 \%-56 \%$ for male athletes, which are considered to be within normal limits and also consistent with the available studies. Kannus (1994) reported that it was not possible to assume an asymmetry between dominant and nondominant side if the bilateral ratio is less than $10 \%$ (Kannus, 1994). In our study, this ratio was determined as $102.00 \pm 15.23,104.15 \pm 21.41$ for all athletes; $102.21 \pm 12.42,102.84 \pm 12.22$ for female athletes and $101.88 \pm 16.90,105.03 \pm 25.78$ for male athletes for flexion and extension, respectively. However, these studies in which the norm values of the bilateral and ipsilateral ratios are established are valid for healthy athletes only. As a result of our study, it can be concluded that there is no ipsilateral and bilateral muscle strength imbalance in athletes, who are deaf. However, we do not think that it would be correct to make a general assumption for all deaf individuals. The reason for the muscle strength values found to be within normal limits could be due to the fact that all deaf individuals are elite athletes. Therefore, there is a need for additional studies to determine the ipsilateral and bilateral muscle strength ratios in deaf individuals, who do not engage in sports.

Static and dynamic core endurance as well as the strength of the athletes were evaluated. The core muscles have important stabilization and mobilization functions, such as providing the neutral spinal alignment, forming the optimal trunk position, and transferring the load in the kinetic chain to the extremities (Huxel Bliven and Anderson, 2013). In our study, the athlete's static core endurance was determined as the ability of the core muscles to maintain the active test position and the dynamic core endurance is determined as how many times the core muscles could perform the primer movements in a specific duration. According to the study of McGill (2010), it was determined that the static core endurance values were $173 \pm 62 \mathrm{sec}$ for the extension endurance test, $134 \pm$ $76 \mathrm{sec}$ for the flexure endurance test, $83 \pm 33 \mathrm{sec}$ for the dominant lateral bridge test and $86 \pm 36 \mathrm{sec}$ for the nondominant lateral bridge test. The relevant timing values were $100.61 \pm 66.83 \mathrm{sec}$; $116.11 \pm 55.34 \mathrm{sec} ; 81.59 \pm 41.47 \mathrm{sec} ; 82.24 \pm 43.41 \mathrm{sec}$, respectively. There were also differences between the genders. Our study also concludes that the static core endurance in deaf athletes is close to the normal limits in terms of lateral core muscles, but the endurance of the anterior and posterior core muscles is significantly lower than that of the findings in McGill's study for deaf athletes. Similarly, in a study that investigates the percentile values of the dynamic core endurance in healthy individuals for various age 
groups, it was determined that the number of repetition of the situp test for an age range of 19-29 years was 30, 40, 57 (25\%, 50\%, $75 \%$, respectively); and repetition for the chest raise test was 35 , 54, 70 (25\%, 50\%, 75\%, respectively) (McIntosh et al., 1998). Our athletes with a mean age of $23.99 \pm 6.56$ years have performed an average $49.29 \pm 12.34$ repetitions of sit ups and $45.53 \pm 18.18$ repetitions of chest raise, which are similar to $75 \%$ percentile values of previous studies. But, when we compered the performance between the genders, we noticed that the female athletes had weaker dynamic core endurance. We could not find any similar studies that compares the dynamic lateral bridge endurance tests between the genders, which were determined in our study. However, when we interpreted our results, we concluded that the lateral flexors, which have relatively lower static endurance compared to the flexor and extensor muscles, were able to perform more repetition than the flexor and extensor muscles. Core muscles provide stabilization and mobilization at the most needed time, thereby protecting the body like a corset and protecting the person from an injury (Huxel Bliven and Anderson, 2013). The low static and relatively dynamic endurance of the anterior and posterior core muscles in deaf athletes may cause these athletes to be vulnerable to injury. For this reason, we think that static endurance training of anterior and posterior core muscles should be added to the training program of deaf athletes, particularly more for male athletes. Besides, all core muscles dynamic endurance training must be added to the female athletes' program.

Balance is also affected in deaf individuals due to the damage in the vestibular apparatus and the disconnect in the relevant nerves (Zebrowska and Zwierzchowska, 2006). For this reason, unlike the other physical fitness parameters, the athlete's hearing impairment directly impacts balance, which at the end highlights the importance of balance evaluation particularly for this group. According to our findings, it was determined that all the athletes' balance with their eyes-open were better than closed regardless of the gender. Since the balance consists of vestibular, visual and proprioceptive components, it was reasonable to assume that balance will deteriorate when we remove the visual component in deaf individuals, who already had one of these components damaged. The functional reach test, which is another subparameter of balance, was also evaluated and it was found that all athletes of both genders could not reach front and back as far as they could in another direction. These results highlight the importance of balance training in deaf athletes and they suggest that these trainings should be performed with different sensory inputs and with proper strategies to especially improve the balance while reaching to the front and back. The single leg balance of the dominant and nondominant sides of the athletes was similar. We think that because there is not any difference between the muscle strength of the dominant and nondominant side, this could be affected to the single leg balance result.

The grip strength is commonly evaluated as it not only reveals the condition of the hand, but also provides information about the strength of the entire upper extremity muscle. Grip strength is important to able to hold a variety of hand-held objects or to tackle competitors during sports activities. There are many studies investigating the normal values for the grip strength. In the metaanalysis of Bohannon et al. (2006), which is composed of many normative studies, the grip strength was determined as $30.6 \mathrm{~kg}$, $27.9 \mathrm{~kg}$ (right and left side, respectively) for females and $53.3 \mathrm{~kg}$, $47.4 \mathrm{~kg}$ (right and left side, respectively) for males aged 20-29. In our study, these values were $24.82 \pm 4.32,22.48 \pm 4.11 \mathrm{~kg}$ (right and left side, respectively) in female athletes and $43.51 \pm$ $7.78,42.47 \pm 7.92 \mathrm{~kg}$ (right and left side, respectively) in the male athletes. The grip strength of the deaf athletes was lower than those in healthy subjects. But in a similar study on adolescents with auditory impairments, the grip strength of sedentary females was found to be lower than male ones (Winnick and Short, 1986).

Anthropometric features play an important role in the selection of athletes and in the performance criteria of the sports (Gutnik et al., 2015). For this reason, different anthropometric features are defined for different sports branches in various studies, but there were no studies that investigated what percentage of fat mass value should exist for athletes, or for deaf individuals. Studies on body composition in deaf individuals were also not available. However, studies suggest that the fat percentage should be $20 \%-$ $25 \%$ for a healthy sedentary female and $15 \%-20 \%$ for a healthy sedentary male. Therefore, as compared to healthy sedentary individuals, it was found that the percentage of body fat of deaf female athletes were similar, whereas deaf male athletes had slightly lower body FM\%. However, we think that studies showing comparison between the nondisabled athletes and unathletic deaf individuals should give more accurate information in this manner.

While interpreting these results, it is necessary to recognize some limitations. There were no control group of sedentary deaf individuals in our study. In addition, it would be possible to enhance our study by adding another group of athletes from different branches without any disabilities.

In conclusion, there is a need for additional studies investigating deaf people and athletes. Therefore, any contributions to the available literature like ours will be very instrumental and it will 
also help to raise awareness. Determination of health-related physical fitness parameters in elite deaf athletes is paramount in increasing the athletic performance and in identifying the most effective training program. In this context, the findings of this study can be used as an initial reference to outline the requirements of the training program for all athletes, who are deaf.

\section{CONFLICT OF INTEREST}

No potential conflict of interest relevant to this article was reported.

\section{REFERENCES}

Akınoğlu B, Kocahan T. Comparison of muscular strength and balance in athletes with visual impairment and hearing impairment. J Exerc Rehabil 2018;14:765-770.

Berckmans K, Maenhout AG, Matthijs L, Pieters L, Castelein B, Cools $\mathrm{AM}$. The isokinetic rotator cuff strength ratios in overhead athletes: assessment and exercise effect. Phys Ther Sport 2017;27:65-75.

Bohannon RW, Peolsson A, Massy-Westropp N, Desrosiers J, Bear-Lehman J. Reference values for adult grip strength measured with a Jamar dynamometer: a descriptive meta-analysis. Physiotherapy 2006; 92:11-15.

Cheung RT, Smith AW, Wong del P. H:Q ratios and bilateral leg strength in college field and court sports players. J Hum Kinet 2012;33:63-71.

Cometti G, Maffiuletti NA, Pousson M, Chatard JC, Maffulli N. Isokinetic strength and anaerobic power of elite, subelite and amateur French soccer players. Int J Sports Med 2001;22:45-51.

Cowan A. Core stability. In: Brukner P, Khan K, editors, Clinical sports medicine. 4th ed. Sydney (Australia): McGraw-Hill; 2012. p. 210-226.

Croisier JL, Ganteaume S, Binet J, Genty M, Ferret JM. Strength imbalances and prevention of hamstring injury in professional soccer players: a prospective study. Am J Sports Med 2008;36:1469-1475.

Dursun OB, Erhan SE, Ibiş EÖ, Esin IS, Keleş S, Şirinkan A, Yörük Ö, Acar E, Beyhun NE. The effect of ice skating on psychological well-being and sleep quality of children with visual or hearing impairment. Disabil Rehabil 2015;37:783-789.

Fischer F, Fink C, Herbst E, Hoser C, Hepperger C, Blank C, Gföller P. Higher hamstring-to-quadriceps isokinetic strength ratio during the first post-operative months in patients with quadriceps tendon compared to hamstring tendon graft following ACL reconstruction. Knee Surg Sports Traumatol Arthrosc 2018;26:418-425.

Ghrairi M, Hammouda O, Malliaropoulos N. Muscular strength profile in Tunisian male national judo team. Muscles Ligaments Tendons J

\section{4;4:149-153.}

Gutnik B, Zuoza A, Zuozienė I, Alekrinskis A, Nash D, Scherbina S. Body physique and dominant somatotype in elite and low-profile athletes with different specializations. Medicina (Kaunas) 2015;51:247-252.

Huxel Bliven KC, Anderson BE. Core stability training for injury prevention. Sports Health 2013;5:514-522.

Jackson AS. The evolution and validity of health-related fitness. Quest 2006;58:160-175.

Kannus P. Isokinetic evaluation of muscular performance: implications for muscle testing and rehabilitation. Int J Sports Med 1994;15 Suppl 1:S11-18

Kurdak SS, Ozgünen K, Adas U, Zeren C, Aslangiray B, Yazıcı Z, Korkmaz S. Analysis of isokinetic knee extension/flexion in male elite adolescent wrestlers. J Sports Sci Med 2005;4:489-498.

Kurková P, Válková H, Scheetz N. Factors impacting participation of European elite deaf athletes in sport. J Sports Sci 2011;29:607-618.

McGill S. Core training: evidence translating to better performance and injury prevention. Strength Cond J 2010;32:33-46.

McIntosh G, Wilson L, Affieck M, Hall H. Trunk and lower extremity muscle endurance: normative data for adults. J Rehabil Outcome Meas 1998:2;20-39.

Nuñez C, Gallagher D, Visser M, Pi-Sunyer FX, Wang Z, Heymsfield SB. Bioimpedance analysis: evaluation of leg-to-leg system based on pressure contact footpad electrodes. Med Sci Sports Exerc 1997;29:524-531.

Stewart DA, Ellis MK. Sports and the deaf child. Am Ann Deaf 2005;150: 59-66.

Stølen T, Chamari K, Castagna C, Wisløff U. Physiology of soccer: an update. Sports Med 2005;35:501-536.

Undheim MB, Cosgrave C, King E, Strike S, Marshall B, Falvey É, Franklyn-Miller A. Isokinetic muscle strength and readiness to return to sport following anterior cruciate ligament reconstruction: is there an association? A systematic review and a protocol recommendation. Br J Sports Med 2015;49:1305-1310.

Vieira LH, de Souza Serenza F, de Andrade VL, de Paula Oliveira L, Mariano FP, Santana JE, Santiago PR. Kicking performance and muscular strength parameters with dominant and nondominant lower limbs in Brazilian elite professional futsal players. J Appl Biomech 2016;32:578585.

Winnick JP, Short FX. Physical fitness of adolescents with auditory impairments. Adapt Phys Activ Q 1986;3:58-66.

Xaverova Z, Dirnberger J, Lehnert M, Belka J, Wagner H, Orechovska K. Isokinetic strength profile of elite female handball players. J Hum Kinet 2015:49:257-266.

Zebrowska A, Zwierzchowska A. Spirometric values and aerobic efficiency of children and adolescents with hearing loss. J Physiol Pharmacol 
2006;57 Suppl 4:443-447.

Zi-Hong H, Lian-Shi F, Hao-Jie Z, Kui-Yuan X, Feng-Tang C, Da-Lang T, Ming-Yi L, Lucia A, Fleck SJ. Physiological profile of elite Chinese female wrestlers. J Strength Cond Res 2013;27:2374-2395.
Ziv G, Lidor R. Vertical jump in female and male volleyball players: a review of observational and experimental studies. Scand J Med Sci Sports 2010;20:556-567. 